\title{
Structure of Electrical Double Layer at Mica/KI Solution Interface
}

\author{
Hiroshi Sakuma ${ }^{1, *}$, Hironori Nakao ${ }^{2}$, Yuichi Yamasaki ${ }^{2}$ and Katsuyuki Kawamura ${ }^{3}$ \\ ${ }^{1}$ Department of Earth and Planetary Sciences, Graduate School of Science and Engineering, Tokyo Institute \\ of Technology, 2-12-1 Ookayama, Meguro-ku, Tokyo 152-8551, Japan \\ ${ }^{2}$ Condensed Matter Research Center and Photon Factroy, Institute of Materials Structure Science, High \\ Energy Accelerator Research Organization, Tsukuba, Ibaraki 305-0801 Japan \\ ${ }^{3}$ Department of Environmental and Civil Engineering, Okayama University, 3-1-1 Tsushima-Naka, Kita-ku, \\ Okayama 7008530, Japan
}

\begin{abstract}
Solid/liquid interfaces control many physical and chemical processes such as electrokinetic phenomena, dispersion of colloidal particles, heteroepitaxial growth of alkali halide, removal of toxic elements in water. The atomic distribution at solid/liquid interfaces is strongly correlated with these properties, and understanding these atomic structures is necessary in order to establish the fundamental physics and chemistry of solid/liquid interfaces. In this study, we investigated the structure of interfaces of mica with aqueous KI solution using surface x-ray scattering. The sub-angstrom-scale electron density profile of the interface is revealed as a function of the distance normal to the interface. The electron density of the KI solution oscillates to remain $10 \AA$ away from the surfaces. The oscillations are interpreted in terms of the adsorbed hydrated $\mathrm{K}^{+}$ions, adsorbed water molecules, and water molecules surrounding the hydrated ions. The adsorbed $\mathrm{K}^{+}$ions are present as inner sphere complexes and the number is enough to compensate the negatively charged mica surface. No significant difference appeared between the surface x-ray scattering profiles of the $\mathrm{KCl}$ and $\mathrm{KI}$ solution interfaces, indicating that their interfacial structures are similar.
\end{abstract}

Keywords: Surface X-ray scattering, Solid-liquid interface, Electron density, Potassium iodide, Molecular dynamics.

\section{INTRODUCTION}

Solid/liquid interfaces control many important physical and chemical processes such as electrokinetic phenomena, dispersion of colloidal particles, heteroepitaxial growth of alkali halide, removal of toxic elements in water, friction and lubrication between solid surfaces. Interfacial double layer theory has been applied for charged solid surfaces to understand and control these physical and chemical processes [1, 2]. The theory is based on the ion distribution near a charged surface and therefore the atomic distribution at solid/liquid interfaces should be revealed to develop the classical double layer theory. On a nanometric scale, the physical properties of liquids could differ from those in bulk due to the liquids' interaction with solid surfaces and the effect of confinement between solid surfaces. Surface forces measurements using surface forces apparatus and atomic force microscopy have revealed the behavior of confined and adsorbed aqueous solutions [1, 3]. Aqueous salt solutions confined between mica surfaces show repulsive hydration forces at a surface separation of $<2 \mathrm{~nm}$ under compression that could not be explained by the classical electrical double layer theory [1]. At these interfaces, continuum

*Address corresponding to this author at the Department of Earth and Planetary Sciences, Graduate School of Science and Engineering, Tokyo Institute of Technology, 2-12-1 Ookayama, Meguro-ku, Tokyo 152-8551, Japan; Tel: +81-3-5734-3722; Fax: +81-3-5734-3538;

E-mail: sakuma.h.aa@m.titech.ac.jp theory cannot be used to understand the interfacial structure and properties of the liquid.

A comparison of molecular dynamics (MD) simulations and X-ray crystal truncation rods (CTR) scattering measurements has suggested the occurrence of excess adsorption of $\mathrm{Cs}^{+}$and $\mathrm{K}^{+}$on a muscovite surface (more than the number needed to compensate for the negatively charged surface) in an aqueous solution [4]. This might be explained by the co-adsorption of counter-ions and co-ions in the solution. Such ion-pair adsorption enhances the maximum number of counter ion that can be adsorbed on a mineral surface; therefore, it is important to understand its mechanism. lons having a large ionic radius generally interact weakly with water molecules and might prefer to form ion-pairs in the solution and adsorb on a mica surface. This hypothesis should be tested systematically for various combinations of counter- and co-ions having different ionic radii.

Here, we investigated the interfacial structure of mica/KI solution on a muscovite surface using X-ray CTR scattering measurements and revealed the structure of electrical double layer. The X-ray CTR scattering technique is a powerful tool for measuring the electron density profile of interfaces with subangstrom resolution $[5,6]$, and it works especially well for mica/aqueous electrolyte solutions [5, 7-12]. A mineral surface yields a rod-shaped X-ray scattering 
perpendicular to the surface called CTR scattering. The shape of the X-ray CTR scattering profile between the Bragg reflection points of the minerals is proportional to the Fourier transform of the electron density distribution around the interface. Therefore, the interfacial electron density distribution can be obtained by analyzing CTR profiles. A comparison of the electron density profiles of the interfaces of mica/ $\mathrm{KCl}$ and $\mathrm{KI}$ solutions might explain the possibility of ion-pair adsorption at the interfaces, because iodide has more electrons than chloride, thereby yielding a large difference in the electron density at the interfaces.

\section{METHODS}

\subsection{X-Ray CTR Scattering Measurements}

Mica (muscovite: $\mathrm{KAl}_{2}(\mathrm{OH})_{2}\left(\mathrm{AlSi}_{3}\right) \mathrm{O}_{10}$ ) belongs to the group of 2:1-layer silicates and consists of an octahedral $\left[\mathrm{AlO}_{6}\right]$ sheet sandwiched between two tetrahedral $\left[(\mathrm{Si}, \mathrm{Al}) \mathrm{O}_{4}\right]$ sheets. One of the four $\mathrm{Si}$ atoms in the tetrahedron is replaced by an $\mathrm{Al}$ atom, thereby causing an overall negative charge on the layers. This charge is compensated for, and the layers are bonded together by an interlayer of $\mathrm{K}^{+}$ions. The lattice constants $(a=5.1919 \AA, b=9.0114 \AA, c=20.0472 \AA$, and $\beta=95.762^{\circ}$ ) and the atomic coordinates of $2 M_{1}$ muscovite used in this study were determined by Rietveld analysis of the powder X-ray diffraction pattern using the RIETAN 2000 [13] program. When mica is immersed in a salt solution, the $\mathrm{K}^{+}$ions on the cleaved surface are easily exchanged with cations [9, 14, 15]. In this study, the surface $\mathrm{K}^{+}$ions would be retained on the surface owing to the presence of a large number of $\mathrm{K}^{+}$ions in the solutions. A concentration of 0.5 molar (M) was chosen because it is sufficiently high to allow the retention of the surface $\mathrm{K}^{+}$ions [14]. Solutions of $\mathrm{KCl}(\mathrm{pH}=5.4)$ and $\mathrm{KI}(\mathrm{pH}=5.4)$ were prepared using pure potassium chloride and potassium iodide powders (Merck, suprapur $>99.99 \%$ ) and pure water having a resistivity of $>18.0 \mathrm{M} \Omega \mathrm{cm}$. The mica crystals were cut into $1 \times 1 \mathrm{~cm}^{2}$ squares and glued onto flat silica glass lenses. The glues were cyano-acrylate or epoxy resin. Fresh, clean (001) mica planes were prepared by peeling. The surface was immediately immersed in a $0.5 \mathrm{M} \mathrm{KCl}$ or $\mathrm{KI}$ solution and kept in it for at least a few hours. Just before the CTR measurements, the mica was mounted on a thin-film liquid cell, which was covered by a $6-\mu \mathrm{m}$ thick polyester film to prevent the solution from evaporating. The X-ray CTR scattering intensities were the integrated intensities by the rocking scans. The intensities were corrected by the scattering area, Lorentz factor, polarization factor, rod interception
[16], and the attenuation factor $\exp (-D \mu / \sin \theta)$ to compensate for attenuation of the X-ray beam through the aqueous solution film. Here $D$ is the film thickness, $\mu$ is the linear absorption coefficient [17] of the aqueous solution and polyester film and $\theta$ is the incident angle of the X-ray beam. The film thickness was determined by comparing the experimentally observed scattering intensities near the Bragg reflection points to theoretical values obtained using the lattice constants measured for the single crystals, because it was difficult to measure the actual liquid thickness in this cell geometry. The $\mathrm{KCl}$ and $\mathrm{KI}$ solutions were determined to be 30 and $40 \mu \mathrm{m}$ thick, respectively. The surface $\mathrm{X}$-ray scattering experiments were performed at the Photon Factory, KEK, Japan (BL-4C) by using monochromatic X-rays of $11.0 \mathrm{keV}$. CTR refers to scattering with a momentum transfer $Q$ vector perpendicular to the interface. The CTR profile was obtained in the range $Q=0.66-4.35 \AA^{-1}$ with individual rocking scans at given $Q$ values. The resolution of the data was approximately $\pi / Q_{\max }$, where $Q_{\max }$ is the highest $Q$ value at which the data were obtained [5]. The resolution of these measurements was $0.72 \AA$.

The electron density profiles of the mica/ $\mathrm{KCl}$ and $\mathrm{KI}$ solution interfaces, which were the Fourier transforms of an atomistic model structure, were obtained by comparing the experimental and calculated CTR profiles. The model structure includes a fixed semiinfinite bulk mica structure, a relaxed surface structure, adsorbed layers of ions and water molecules, and bulk water above the interface. To reproduce the experimental CTR profile, structural relaxation of the mica surface was performed at 0 to $-30 \AA$ from the interface. The electron densities of the adsorbed layers and bulk water were represented by the superposition of Gaussian distributions. Fitting was performed using a least-squares method applied by an ANA-ROD program [18] with a few modifications in order to incorporate the structure factor of liquid. The roughness of the mica surface was not corrected in the fitting procedures.

The fittings results were evaluated using the $x^{2}$ and $R$-factor values, which were calculated as,

$$
\begin{aligned}
& \chi^{2}=\frac{1}{n} \sum_{j=1}^{n} \frac{\left[\left|F_{j}\right|-\left|F_{\mathrm{fit}, j}\right|\right]^{2}}{\sigma_{j}^{2}}, \\
& R=\frac{\sum_{j=1}^{n}|| F_{j}|-| F_{\mathrm{fit}, j}||}{\sum_{j=1}^{n}\left|F_{j}\right|} .
\end{aligned}
$$


Here $n$ is the total number of data points; $F_{j}$ and $\sigma_{j}$ are the structure factor and statistical uncertainty [19] of the $j$-th data point, respectively; and $F_{\text {fit, } j}$ is the structure factor calculated using an atomistic model structure. Typical $X^{2}$ values are $\sim 1$ to $\sim 5$ for the best-fit structures of high-resolution X-ray CTR data [5].

\section{RESULTS AND DISCUSSION}

\subsection{X-Ray CTR Scattering Profiles}

The measured X-ray CTR scattering profiles are shown in Figure 1 together with the best-fit models of the interfacial structure. The vertical axis was converted to the specular reflectivity to facilitate comparison with a previous result [9] for a $0.5 \mathrm{~m} \mathrm{KCl}$ solution on a mica surface. The error bars indicate that the sum of statistical uncertainties [19] and errors due to the background definition for the integration of intensities. The high intensities of the CTR profile at $Q$ $\approx 0.6,1.3,1.9,2.5,3.1,3.8$, and $4.4 \AA^{-1}$ correspond to the Bragg reflection points of mica $(00 /$, where $I=2,4$, $6,8,10,12$, and 14) planes. The shape of the CTR profile between the Bragg points provides structural information on the mica/potassium halide solution interfaces. The intensities ranged from $10^{-6}$ next to the Bragg points to $10^{-11}$ at $Q=2.6$ and $3.7 \AA^{-1}$. As shown in the figure, no significant difference was observed within the error bars among the profiles of the previously reported $0.5 \mathrm{~m} \mathrm{KCl}$ solution [9], and the 0.5 $\mathrm{M} \mathrm{KCl}$ and $\mathrm{KI}$ solutions measured in this study.

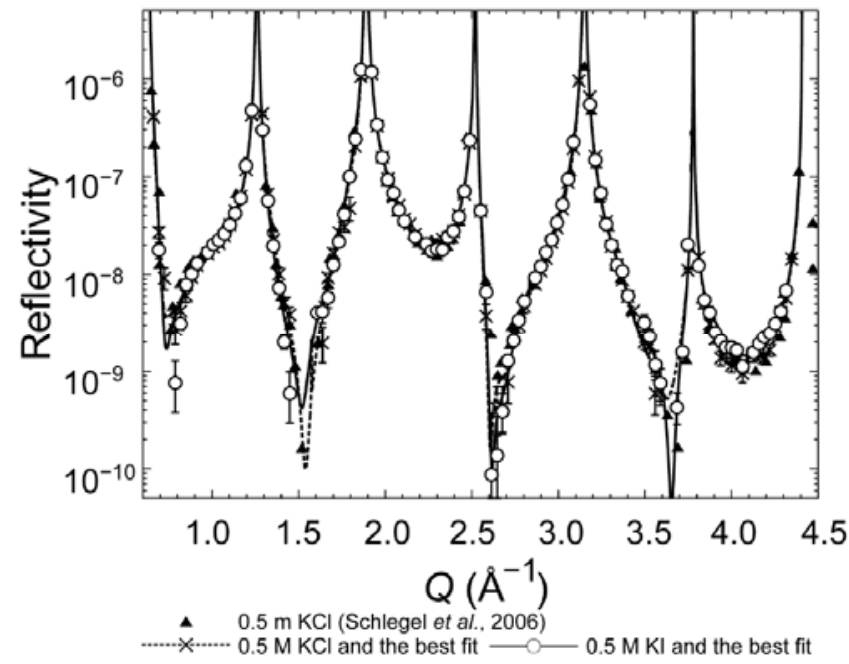

Figure 1: Comparison of X-ray CTR scattering profiles for mica/0.5 M KCl (crosses) and $0.5 \mathrm{M} \mathrm{KI}$ (open circles) solution interfaces. Vertical axis represents the specular reflectivity of the interfaces. Previous result for $0.5 \mathrm{~m} \mathrm{KCl}$ solution on a mica surface [9] is shown for comparison. Dashed and solid lines indicate the profiles calculated by a best-fit model to mica $/ 0.5 \mathrm{M} \mathrm{KCl}\left(X^{2}=2.92, R\right.$-factor $\left.=0.048\right)$ and mica/ $0.5 \mathrm{M}$ $\mathrm{KI}$ solution $\left(x^{2}=5.79, R\right.$-factor $\left.=0.053\right)$ interfaces, respectively.

\subsection{Electron Density Profiles at the Mica/Potassium Halide Solution Interfaces}

The $X^{2}$ and $R$-factor values of the best-fit models shown in Figure 1 were $X^{2}=2.92$ and 0.048 for the mica/0.5 M KCl interface and $x^{2}=5.79$ and 0.053 for the mica/0.5 M KI interface. We obtained resolutionbroadened electron density profiles (Figures 2 and 3 ) as a result of the finite resolution arising from the limited range of the CTR scattering data. The broadening procedure was performed as described in the literature [5]. The origin of the horizontal axis is the location of the outermost oxygen layer of the mica surface.

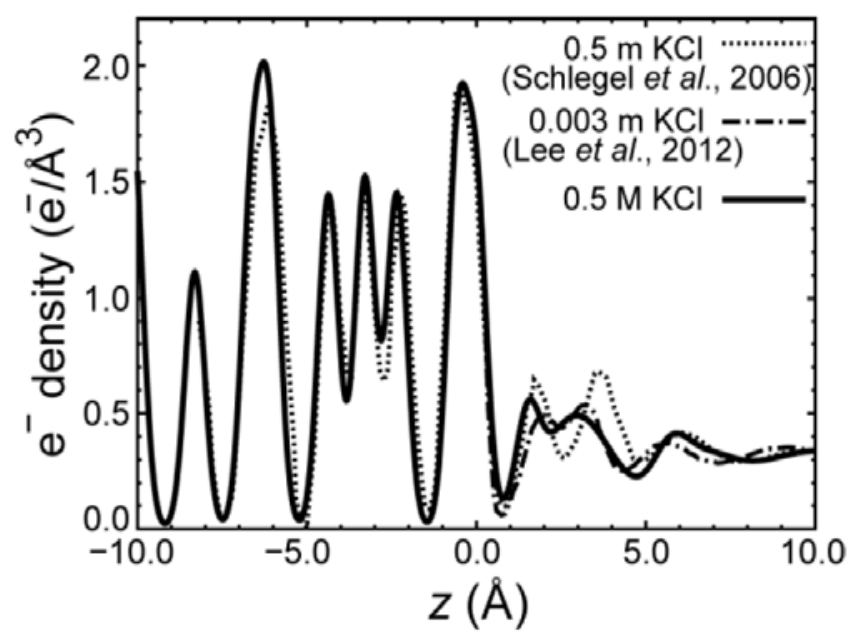

Figure 2: Comparison of electron density profiles of mica $/ \mathrm{KCl}$ solution interfaces with different salt concentrations. Solid line is the result of this study for a $0.5 \mathrm{M} \mathrm{KCl}$ solution. Dotted and dashed lines indicate the results for $0.5 \mathrm{~m} \mathrm{KCl} \mathrm{[9]} \mathrm{and} 0.003$ $\mathrm{m} \mathrm{KCl}$ solutions [12], respectively. Origin of the $z$ axis is the position of the outermost oxygen layer of the mica surface.

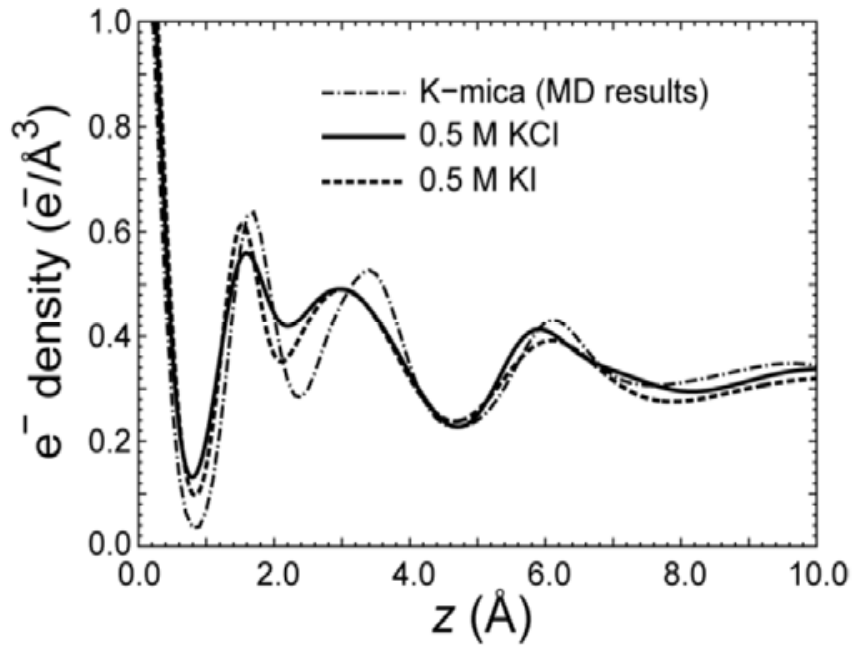

Figure 3: Comparison of electron density profiles in the liquid areas of mica $/ 0.5 \mathrm{M} \mathrm{KCl}$ and mica/0.5 M KI solution interfaces with the corresponding MD simulations $[4,20]$. 


\subsubsection{Mica/KCl Solutions}

First, we check the reliability of our measurements by comparing them with previous measurements. $\mathrm{Mica} / \mathrm{KCl}$ solution interfaces have been measured for several different salt concentrations [9, 12], as shown in Figure 2. The result of our study $(0.5 \mathrm{M} \mathrm{KCl})$ exhibited an electron density oscillation in the liquid at $0.0<z<10.0 \AA$. Three large peaks were observed at $z$ $=1.6,3.0$, and $5.9 \AA$. The number of peaks their positions are similar with the previous results for 0.003 $\mathrm{m} \mathrm{KCl} \mathrm{[12]} \mathrm{and} 0.5 \mathrm{~m} \mathrm{KCl}$ [9]. The sum of the integrated electron density for the first $(z=1.6 \AA)$ and second $(z=3.0 \AA)$ solution peaks were compared with the previous $0.5 \mathrm{~m} \mathrm{KCl}$ in Table 1. The integrated electron density was agreed with in the error.

Table 1: Integrated Electron Densities at the Liquid Area of Muscovite $/ 0.5 \mathrm{M} \mathrm{KCl}$ and $0.5 \mathrm{M} \mathrm{KI}$ Interface. The Results of Previous Experiments [9] and MD Simulations $[4,20]$ are Shown for Comparison

\begin{tabular}{|c|c|}
\hline $\begin{array}{c}\text { Integrated electron density } \\
\left(\mathbf{e}^{-} / \AA^{2}\right)\end{array}$ & $\begin{array}{c}\text { Sum of the first and second } \\
\text { peaks }\end{array}$ \\
\hline \hline $0.5 \mathrm{M} \mathrm{KCl}$ & 1.56 \\
\hline $0.5 \mathrm{~m} \mathrm{KCl}[9]$ & $1.77 \pm 0.26$ \\
\hline $0.5 \mathrm{M} \mathrm{KI}$ & 1.57 \\
\hline $\mathrm{MD}[4,20]$ & 1.40 \\
\hline
\end{tabular}

The difference of the peak positions among the previous data for a $0.5 \mathrm{~m} \mathrm{KCl}$ solution [9] and our data might be the measured small range of $Q$ values (0.66 $4.35 \AA^{-1}$ ) in this study compared to the previous study $\left(0.25 \sim 5.0 \AA^{-1}\right)$ and the result of different modeling and fitting procedures, as suggested by the high $x^{2}$ value (15.6) of the previous study, because there was no significant difference among the X-ray CTR profiles within the error bars as shown in Figure 1.

The peak height, positions, and periodicity of our data were almost the same as the results of our previous MD simulations [4, 20], as shown in Figure 3. In the MD simulations, "K-mica" denotes a condition in which many $\mathrm{K}^{+}$ions are present in the water, indicating that this condition corresponds to a highly concentrated salt solution in contact with a mica surface. The MD results could be a plausible model for explaining the species adsorbed on the interface. The first maximum in the simulations at $z=1.7 \AA$ was explained as adsorbed potassium ions and water molecules; the second peak at $z=3.4 \AA$ represented hydrated water molecules around the $\mathrm{K}^{+}$ions; and the third peak at $z=$ $6.1 \AA$ was the subsequent density oscillation of water.

\subsubsection{Mica/KI Solution}

The obtained electron density profile for the mica/0.5 M KI solution interface is shown in Figure 3. As we determined qualitatively by comparing the X-ray CTR profiles of the $\mathrm{KCl}$ and $\mathrm{KI}$ solutions, the peak positions and heights were almost the same for both solutions. The integrated electron densities are shown in Table 1 as sums of the first and second peaks. If many iodide ions were present near the surface, the electron density should be larger than that for the $\mathrm{KCl}$ solution because of the large number of electrons in iodide relative to that in chloride ions. The integrated densities of the $0.5 \mathrm{M} \mathrm{KCl}$ and $\mathrm{KI}$ solutions were almost equal indicating that potassium-halide ion-pair adsorption on a negatively charged mica surface cannot be stressed only by the x-ray CTR scattering method. The slightly smaller value obtained in the MD simulation might be attributable to the absence of the halide ions in the simulations. In future, the difference should be discussed in detail using simulations that include halide ions.

\subsection{Implications for the Electrical Double Layers of Potassium Halide Solutions on a Mica Surface}

The comparison of specular x-ray CTR measurements and previous MD simulations revealed that the electrical double layers of $\mathrm{KI}$ solution on a mica surface. The electron density oscillations of $\mathrm{KI}$ solution perpendicular to a mica surface correspond to the adsorbed $\mathrm{K}^{+}$ions, hydrated water molecules around $\mathrm{K}^{+}$ ions, and surrounding water molecules. The $\mathrm{K}^{+}$ions would be present as inner sphere complexes on a mica surface and the number of adsorbed $\mathrm{K}^{+}$ions as inner sphere complexes is enough to compensate the negatively charged mica surface. We had expected that considerable ion-pair adsorption might occur on a mica surface in the $\mathrm{KI}$ solution. The experimental results in this study demonstrated that potassiumhalide pair adsorption on a mica surface should be minor even in presence of weakly hydrated iodide ions in the solution. However the ion-pair adsorption should be carefully investigated in the future by using a combination of different experimental and theoretical approaches.

\section{CONCLUSIONS}

In this study, we revealed the sub-angstrom-scale structure of mica/KI solution interface by using X-ray CTR scattering measurements. The electron density of the $\mathrm{KI}$ solution observed using these measurements oscillated to remain at $10 \AA$ from the surface. A 
comparison with previous MD simulations indicates that the oscillation corresponds to hydrated $\mathrm{K}^{+}$ions, adsorbed water molecules, and water molecules surrounding the hydrated ions. The adsorbed $\mathrm{K}^{+}$ions are present as inner sphere complexes and the number is enough to compensate the negatively charged mica surface. No significant difference was observed in the interfacial densities of the $\mathrm{KCl}$ and $\mathrm{KI}$ solutions. The results imply that potassium-halide ion-pair adsorption on a negatively charged mica surface is minor even in presence of iodide anions, which interact weakly with water molecules.

\section{ACKNOWLEDGEMENT}

This research was partially supported by a Grant-inAid for Young Scientists (B) (23740390) from the Ministry of Education, Culture, Sports, Science and Technology (MEXT), Japan.

\section{REFERENCES}

[1] Israelachvili JN. Intermolecular and surface forces. $2^{\text {nd }}$ ed. New York: Academic Press 1992.

[2] Butt H-J, Graf K, Kappl M. Physics and chemistry of interfaces. Weinheim: Wiley-Vch Verlag GmbH \& Co. KGaA 2003.

[3] Butt H-J, Cappella B, Kappl M. Forces measurements with the atomic force microscope: Technique, interpretation and applications. Surf Sci Rep 2005; 59: 1-152.

http://dx.doi.org/10.1016/j.surfrep.2005.08.003

[4] Sakuma H, Kawamura K. Structure and dynamics of water on $\mathrm{Li}^{+}-, \mathrm{Na}^{+}-, \mathrm{K}^{+}-, \mathrm{Cs}^{+}-, \mathrm{H}_{3} \mathrm{O}^{+}$-exchanged muscovite surfaces: A molecular dynamics study. Geochim Cosmochim Acta 2011; 75: 63-81.

http://dx.doi.org/10.1016/j.gca.2010.10.007

[5] Fenter P. X-ray reflectivity as a probe of mineral-fluid interfaces: A user guide. Rev Mineral Geochem 2002; 49: 149-20.

http://dx.doi.org/10.2138/gsrmg.49.1.149

[6] Willmott P. An introduction to synchrotron radiation: Techniques and applications. John Wiley \& Sons, Ltd. 2011. http://dx.doi.org/10.1002/9781119970958

[7] Cheng L, Fenter P, Nagy KL, Schlegel ML, Sturchio NC. Molecular-scale density oscillations in water adjacent to a mica surface. Phys Rev Lett 2001; 87: 156103. http://dx.doi.org/10.1103/PhysRevLett.87.156103

[8] Fenter P, McBride MT, Srajer G, Sturchio NC, Bosbach D. Structure of barite (001)- and (210)-water interfaces. J Phys Chem B 2001; 105: 8112-9.

http://dx.doi.org/10.1021/jp0105600
[9] Schlegel ML, Nagy KL, Fenter P, Cheng L, Sturchio NC, Jacobsen SD. Cation sorption on the muscovite (001) surface in chloride solutions using high-resolution x-ray reflectivity. Geochim Cosmochim Acta 2006; 70: 3549-65. http://dx.doi.org/10.1016/j.gca.2006.04.011

[10] Park C, Fenter PA, Nagy KL, Sturchio NC. Hydration and distribution of ions at the mica-water interface. Phys Rev Lett 2006; 97: 016101 http://dx.doi.org/10.1103/PhysRevLett.97.016101

[11] Sakuma H, Kondo T, Nakao H, Shiraki K, Kawamura K. Structure of hydrated sodium ions and water molecules adsorbed on the mica/water interface. J Phys Chem C 2011; 115: 15959-64.

http://dx.doi.org/10.1021/jp111936s

[12] Lee SS, Fenter P, Nagy KL, Sturchio NC. Monovalent ion adsorption at the muscovite (001)-solution interface: Relationships among ion coverage and speciation, interfacial water structure, and substrate relaxation. Langmuir 2012; 28 : 8637-50. http://dx.doi.org/10.1021/la300032h

[13] Izumi F, Ikeda T. A rietveld-analysis program Rietan-98 and its applications to zeolites. Mater Sci Forum 2000; 321-324: 198-205.

http://dx.doi.org/10.4028/www.scientific.net/MSF.321$\underline{324.198}$

[14] Pashley RM. DLVO and hydration forces between mica surfaces in $\mathrm{Li}+, \mathrm{Na}+, \mathrm{K}+$, and $\mathrm{Cs}+$ electrolyte-solutions - a correlation of double-layer and hydration forces with surface cation-exchange properties. J Coll Interf Sci 1981; 83: 53146. http://dx.doi.org/10.1016/0021-9797(81)90348-9

[15] Bowers GM, Bish DL, Kirkpatrick RJ. Cation exchange at the mineral-water interface: $\mathrm{H}_{3} \mathrm{O}^{+} / \mathrm{K}^{+}$competition at the surface of nano-muscovite. Langmuir 2008; 24: 10240-4. http://dx.doi.org/10.1021/la8021112

[16] Vlieg E. Integrated intensities using a six-circle surface x-ray diffractometer. J Appl Crystallogr 1997; 30: 532-43. http://dx.doi.org/10.1107/S0021889897002537

[17] Chantler CT. Theoretical form factor, attenuation and scattering tabulation for $z=1-92$ from $e=1-10 \mathrm{eV}$ to $e=0.4$. $1.0 \mathrm{meV}$. J Phys Chem Ref Data 1995; 24: 71-643. http://dx.doi.org/10.1063/1.555974

[18] Vlieg E. Rod: A program for surface x-ray crystallography. J Appl Crystallogr 2000; 33: 401-5. http://dx.doi.org/10.1107/S0021889899013655

[19] Bevington PR, Robinson DK. Data reduction and error analysis for the physical sciences. New York: McGraw-Hill 2003.

[20] Sakuma H, Kawamura K. Structure and dynamics of water on muscovite mica surfaces. Geochim Cosmochim Acta 2009; 73: 4100-10.

http://dx.doi.org/10.1016/j.gca.2009.05.029

(C) 2012 Sakuma et al.; Licensee Lifescience Global.

This is an open access article licensed under the terms of the Creative Commons Attribution Non-Commercial License (http://creativecommons.org/licenses/by-nc/3.0/) which permits unrestricted, non-commercial use, distribution and reproduction in any medium, provided the work is properly cited. 